\title{
Optical Character Recognition using Artificial Intelligence
}

\author{
Shreshtha Garg \\ CS Department \\ Shri Ramswaroop Memorial \\ University, Lucknow
}

\author{
Kapil Kumar Gupta \\ CS Department \\ Shri Ramswaroop Memorial \\ University, Lucknow
}

\author{
Nikhil Prabhakar \\ CS Department \\ Shri Ramswaroop Memorial \\ University, Lucknow
}

\author{
Amulya Ratan Garg \\ CS Department \\ Shri Ramswaroop Memorial University, Lucknow
}

\author{
Aayush Trivedi \\ CS Department \\ Shri Ramswaroop Memorial University, Lucknow
}

\begin{abstract}
This is a complete Optical Recognition System using artificial intelligence. In this paper we have dealt with words and character detection. The OCR system that will train itself and help in extracting text from any image by using neural networks and back propagation techniques. Earlier we had to store data in a DATABASE while performing operations but with this we will be able to train our systems. We will have to store only a limited amount of data and the software will self train itself for future entries.
\end{abstract}

\section{Keywords}

OCR, Artificial Intelligence, Neural Network. Feature extraction.

\section{INTRODUCTION}

Optical Character Recognition, as the name suggests deals with the conversion of pictures that may be handwritten, typed or printed text into machine encoded text electronically on a scanned document, an image of a document with subtitles. OCR is a field of research in pattern recognition, artificial intelligence and computer vision. As far as the document input tasks are concerned, this proves to be a very cost effective and faster method that helps us to free large amount of spaces. Character recognition of printed material is itself a challenging problem since there is a variation of the same character due to type of fonts, writing format of characters or types of noises[1].It helps in reducing human errors up to a definite limit. As the size and shape of fonts is not the same for all the places it becomes very difficult to recognize the characters. To reduce this problem pre-processing, feature extraction and recognition techniques must be error free. Sometimes the image acquires noise due to electrical distortion, signal change or scanning techniques which distorts the characters and makes it very difficult to extract features. Therefore, a good character recognition approach must eliminate the noise after reading binary picture data, smooth the picture for better recognition, extract features efficiently, train the system and classify patterns.

This paper will tell us that how artificial neural network will help us in increasing the performance of OCR and enable us in recognizing different characters using different datasets and training sets. A neural network is a powerful data processing tool that is able to capture and represent complex input/output relationships. The motivation for the development of neural network technology stemmed from the desire to develop an artificial intelligent system software that could perform "intelligent" tasks similar to those performed by the human brain. Neural networks resemble the human brain in the following two ways: they acquire knowledge through previous learning, and the knowledge is stored within interneuron connection strengths known as synaptic weights [1], [2].

Various techniques are used for designing an efficient OCR system. Some of them are Matrix Matching, Fuzzy Logic, Feature Extraction, Structural Analysis, Bayesian Classification and Neural Network. In Matrix Matching, an image is compared to a stored glyph on a pixel-by-pixel basis; it is also known as "pattern matching. This relies on the input glyph being correctly isolated from the rest of the image, and on the stored glyph being in a similar font and at the same scale. This technique works best with typewritten text and does not work well when new fonts are encountered[3]. Fuzzy logic is a multi-valued logic that allows intermediate values to be defined between conventional evaluations like yes/no, true/false, black/white etc [4]. Feature extraction starts from an initial set of measured data and builds derived values (features) intended to be informative and non-redundant, facilitating the subsequent learning and generalization steps, and in some cases leading to better human interpretations [5]. Structural Analysis identifies characters by examining their sub features- shape of the image, sub-vertical and horizontal histograms. Its character repair capability is great for low quality text and newsprints [4].And finally the neural network that is explained above.

In this project we will deal with both training and extraction of text from images that would help people in real time and work with huge amount of data easily. By using this application children would be able to learn pronunciations and improve their reading skills as the text gets converted to speech. The specially disabled people would be able to gain information and learn by listening. The process of OCR contains steps including segmentation, feature extraction, and classification. The reason behind the development of the technique is the advent of Unicode and support of complete scripts on computer. The system components proposed includes, 1)Image Scanning, 2)Image Segmentation, 3)Noise Removal 4)Feature Extraction 5)OCR Post Processing and 6)Output from the image in proper Format.

\section{PREVIOUS WORKS}

Character recognition may be a set of pattern recognition space. to duplicate human functions by machines associate degreed creating the machine perform common tasks like 
reading is an ancient dream. The origins of character recognition dates back to 1870 when C.R.Carey of Boston Massachusetts $[6,7,8]$ fabricated tissue layer that was a picture transmission system using a mosaic of photocells. The history of OCR are often derived as early as 1900, when the Russian scientist Tyuring trying to develop an aid for the visually handicapped [9]. The first character recognizers appeared in the middle of the 1940s with the development of digital computers [6].

After 20 years Nipkow [15] developed sequential scanner which was a major breakthrough both for modern television and reading machines. During the first few decades of Nineteen century several attempts were made to develop devices to aid the blind through experiments with OCR [8].

By 1950 the technological revolution [6,9] was moving forward at very fast speed and e-data processing was become an upcoming and important field. The commercial character recognizers available in $1950 \mathrm{~s}$ where electronic tablets captured the $\mathrm{x}-\mathrm{y}$ coordinate data of pen tip movement were first introduced. This innovation enabled the researchers to work on the online handwriting recognition problem [6]. The data entry was performed through punched cards. A cost effective way of handling the increasing amount of data was then required. At the same time the technology for machine reading was becoming sufficiently mature for application. By mid 1950s OCR machines became commercially available [8].The first OCR reading machine [16] was installed at Reader's Digest in 1954. This equipment was used to convert typewritten sales reports into punched cards for input into the computer.

In 1966 a thorough study of OCR requirements was completed and an American standard OCR character set was defined as OCR-A. This font was highly stylized and designed to facilitate optical recognition although still readable to humans. An European font was also designed as OCR-B that had more natural fonts than American standard. An Attempt was made to merge two fonts in one standard through machines which could read both standards.

Actual progress on OCR systems achieved during 1990s using the new development tools and methodologies which are empowered by the continuously growing information technologies. In the early 1990s, image processing and pattern recognition techniques were efficiently combined with artificial intelligence methodologies. Researchers developed complex OCR algorithms, which receive high-resolution input data and require extensive number crunching in the implementation phase. Now a days, in addition to the more powerful strong computers and more accurate electronic tools such as scanners, cameras, and electronic tablets, we have efficient, modern use of methodologies such as artificial neural networks(ANNs), hidden Markov models (HMMs), fuzzy set reasoning and natural language processing. The recent systems for the machine printed offline [17, 6] and limited vocabulary, user dependent online handwritten characters [17] are quite satisfactory for restricted applications. However, still a long way to go in order to reach the ultimate goal of machine simulation of fluent human reading especially for unconstrained online and offline handwriting.

Rahul KALA and team proposed work on Offline Handwriting Recognition method with used Genetic Algorithm. In this research describe to a piece of paper and then convert it into text. They are used genetic algorithm to implement Offline Handwriting Recognition [10]. Brandon
Maharrey COMP 6600 Artificial Intelligence Spring 2009 they survey about A Neural Network Implementation of Optical Character Recognition that measure that neural network is also use in OCR for the handwritten notes or words [11]. Sang Sung Park, Won Gyo Jung, Young et.al: teams they are implemented Optical Character Recognition System Using BP Algorithm they told her They use OCR (OCR: Optical Character Recognition) technique which is that saving relevant documents to DB after extracting text by using OCR. That is, text should be entered to DB after classifying segments one by one in realized whole document after doing character recognition through OCR. In this paper, in order to solve this problem, we constructed OCR system that saves abstracted characters to DB automatically after extracting only equivalent and necessary characters from a large amount of documents by using BP algorithm [12]. Made Edwin Wira Putra, Iping Supriana Suwardi both has implement the Structural off-line handwriting character recognition using the purpose of those model is to give the ability in improving recognition accuracy without relying in normalization technique. They are use in graph technique. The graph consists of several edges that indicate the connected vertices. The vertices are joining and to form a curve that make the character. The curve is extracted by analyzing the character's chain code, and its string feature is created using some principle [13]. Krupa dholakia has to define about the handwritten character recognition technique are divided into some subparts such as preprocessing, segmentation, feature extraction, classification and post processing[14].

Dr.Mrs.V.V.Patil, Rajharsh Vishnu Sanap, Rohini Babanrao Kharate carried out a study handwritten character recognition using Artificial Neural Network. Artificial neural networks are commonly used to perform character recognition due to their high noise tolerance. The systems have the ability to yield excellent results. The feature extraction step of optical character recognition is the most important. A poorly chosen set of features will yield poor classification rates by any neural network. A simplistic approach for recognition of Optical characters using artificial neural networks has been described[20]. Sanjay Kumar, Narendra Sahu , Aakash Deep, Khushboo Gavel, Miss Rumi Ghosh have introduce the offline handwriting recognition, to provide in improving the ability of recognition accuracy. In this they used the neural network method to implement the OCR. They had researched the Indian doctor prescription and decided to implement the OCR in medical point of view. In this method unknown word (i.e. handwritten word) is input and recognition text is output [19]. Chowdhury Md Mizan, Tridib Chakraborty and Suparna Karmakar present a brief survey of the applications in various fields along with experimentations into few selected fields. The proposed method is very much efficient to extract all kinds of bimodal images including blur and illumination. The paper will act as a good literary survey for researchers starting to work in the field of Optical Character Recognition. [18]

\section{PROPOSED WORK}

In this we have used Optical Character Recognition with Neural Network. Our work starts with the acquisition of a given image and then scanning the given image for editing and producing desirable outputs. We then pre-processed our images and applied segmentation on it so that the text could be separated. After this the features of the image were extracted which were then followed by recognition and classification. 


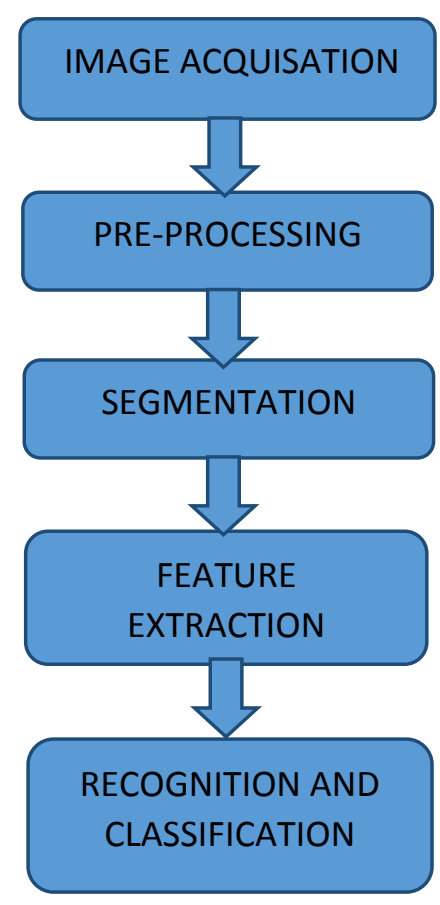

Fig1.Components of OCR with Neural Network

The detailed process is listed below:

\subsection{Acquisition of image}

In this we read or scan the image and produce a digital image. We do this with the help of an optical scanner that converts light intensity into gray scale.

\subsection{Pre-Processing}

In this we aim to reduce the color or noise in an image. The scanned image might have some noise which needs to be improved for making the recognition of characters easy and efficient. This is done by applying smoothing on the scanned image and reducing the background noise, like for salt and pepper we use the median filter for smoothing.

\subsection{Segmentation}

In this process we try to binaries the image for contrasting the text with the background. It is one of the major steps in OCR and with good segmentation we can improve the working of OCR. In this we basically extract the main components of our image i.e. the text that we further use for recognition. It can be of three types - line, word or character segmentation.

\subsection{Feature Extraction}

In this the important features of the symbols are extracted leaving out the unimportant attributes. We aim to extract the connected pixels. The features can be extracted using different techniques like the distribution of points, transformations and series expansions and structural analysis of characters.

\subsection{Recognition and Classification}

In this we try to test the features with our existing dataset. If the features match the result is displayed else we update the database with that feature. For classification we use the ANN approach. The data is classified based on the mean distance, spatial position of pixel and pixel value. Here, we use apply training and recognition using the back propagation technique.
4. RESULT ANALYSIS

Table1: Input image size with luminance

\begin{tabular}{|c|c|}
\hline SIZE IN BYTES & LUMINANCE \\
\hline 24542 & 118.94 \\
\hline 328580 & 144.54 \\
\hline 479960 & 149.33 \\
\hline 389360 & 150.57 \\
\hline 467910 & 105.79 \\
\hline 905080 & 134.03 \\
\hline 971820 & 122.67 \\
\hline 808690 & 111.61 \\
\hline 319560 & 175.42 \\
\hline 360820 & 64.238 \\
\hline 750670 & 134.33 \\
\hline 290310 & 147.97 \\
\hline 613000 & 101.68 \\
\hline 142640 & 169.24 \\
\hline 1930600 & 142.59 \\
\hline 154900 & 191.37 \\
\hline 92354 & 157.38 \\
\hline 168730 & 101.17 \\
\hline 221630 & 158.88 \\
\hline 117950 & 39.775 \\
\hline 85293 & 132.3 \\
\hline 86947 & 17.815 \\
\hline 78944 & 123.04 \\
\hline 111400 & 106.72 \\
\hline 77248 & 28.036 \\
\hline 39662 & 216.57 \\
\hline 141980 & 231.75 \\
\hline 72648 & 237.33 \\
\hline 33480 & 171.38 \\
\hline 77815 & 193.75 \\
\hline 17383 & 150.97 \\
\hline 17683 & 208.45 \\
\hline 18894 & 146.41 \\
\hline 5873 & 86.323 \\
\hline 43415 & 180.37 \\
\hline 10560 & 168.92 \\
\hline 39662 & 216.57 \\
\hline 337520 & 140.08 \\
\hline
\end{tabular}




\begin{tabular}{|l|l|}
\hline 11155 & 49.519 \\
\hline 8918 & 40.561 \\
\hline
\end{tabular}

Variation in size and luminance

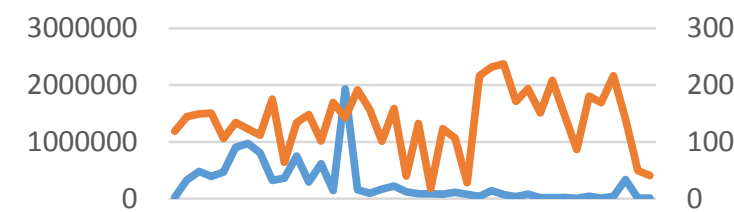

1471013161922252831343740

SIZE IN BYTES LUMINANCE

Fig2. Comparison between size of image and luminance

Table2: luminance of images with recognition time

\begin{tabular}{|c|c|}
\hline LUMINANCE & TIME IN MILLIS \\
\hline 118.94 & 74.229 \\
\hline 144.54 & 133.73 \\
\hline 149.33 & 218.47 \\
\hline 150.57 & 865.6 \\
\hline 105.79 & 606.84 \\
\hline 134.03 & 430.09 \\
\hline 122.67 & 914.51 \\
\hline 111.61 & 461.63 \\
\hline 175.42 & 254.42 \\
\hline 64.238 & 224.55 \\
\hline 134.33 & 9833.5 \\
\hline 147.97 & 228.6 \\
\hline 101.68 & 1400.9 \\
\hline 169.24 & 63.856 \\
\hline 142.59 & 3268.5 \\
\hline 191.37 & 207.24 \\
\hline 157.38 & 89.037 \\
\hline 101.17 & 98.947 \\
\hline 158.88 & 471.62 \\
\hline 39.775 & 90.419 \\
\hline 132.3 & 75.694 \\
\hline 17.815 & 30.867 \\
\hline 123.04 & 29.908 \\
\hline 106.72 & 78.36 \\
\hline 28.036 & 18.568 \\
\hline
\end{tabular}

\begin{tabular}{|l|l|}
\hline 216.57 & 833.07 \\
\hline 231.75 & 2426.2 \\
\hline 237.33 & 1251 \\
\hline 171.38 & 488.79 \\
\hline 193.75 & 466.11 \\
\hline 150.97 & 232.93 \\
\hline 208.45 & 428.83 \\
\hline 146.41 & 327.36 \\
\hline 86.323 & 71.843 \\
\hline 180.37 & 196.2 \\
\hline 168.92 & 266.31 \\
\hline 216.57 & 831.62 \\
\hline 140.08 & 3278.6 \\
\hline 49.519 & 40.708 \\
\hline 40.561 & 24.9 \\
\hline & \\
\hline
\end{tabular}

\section{Variations in luminance with time}

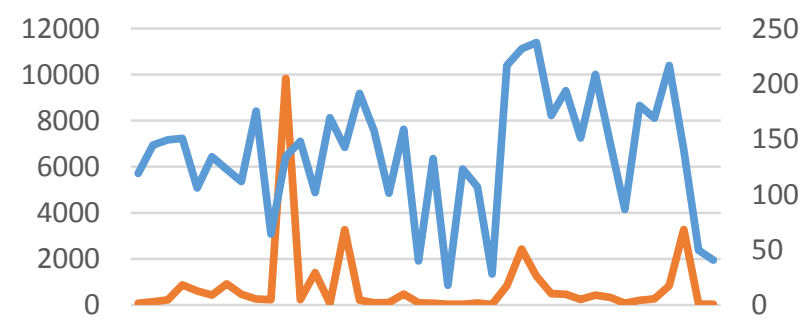

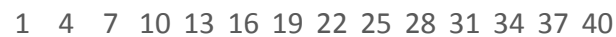

—TIME IN MILLIS — LUMINANCE

Fig3. Comparison between luminance and character recognition time

Table 3: Size of images with recognition time

\begin{tabular}{|l|l|}
\hline SIZE IN BYTES & TIME IN MILLIS \\
\hline 24542 & 74.229 \\
\hline 328580 & 133.73 \\
\hline 479960 & 218.47 \\
\hline 389360 & 865.6 \\
\hline 467910 & 606.84 \\
\hline 905080 & 430.09 \\
\hline 971820 & 914.51 \\
\hline 808690 & 461.63 \\
\hline 319560 & 254.42 \\
\hline 360820 & 224.55 \\
\hline
\end{tabular}




\begin{tabular}{|c|c|}
\hline 750670 & 9833.5 \\
\hline 290310 & 228.6 \\
\hline 613000 & 1400.9 \\
\hline 142640 & 63.856 \\
\hline 1930600 & 3268.5 \\
\hline 154900 & 207.24 \\
\hline 92354 & 89.037 \\
\hline 168730 & 98.947 \\
\hline 221630 & 471.62 \\
\hline 117950 & 90.419 \\
\hline 85293 & 75.694 \\
\hline 86947 & 30.867 \\
\hline 78944 & 29.908 \\
\hline 111400 & 78.36 \\
\hline 77248 & 18.568 \\
\hline 39662 & 833.07 \\
\hline 141980 & 2426.2 \\
\hline 72648 & 1251 \\
\hline 33480 & 488.79 \\
\hline 77815 & 466.11 \\
\hline 17383 & 232.93 \\
\hline 17683 & 428.83 \\
\hline 18894 & 327.36 \\
\hline 5873 & 71.843 \\
\hline 43415 & 196.2 \\
\hline 10560 & 266.31 \\
\hline 39662 & 831.62 \\
\hline 337520 & 3278.6 \\
\hline 11155 & 40.708 \\
\hline 8918 & 24.9 \\
\hline
\end{tabular}

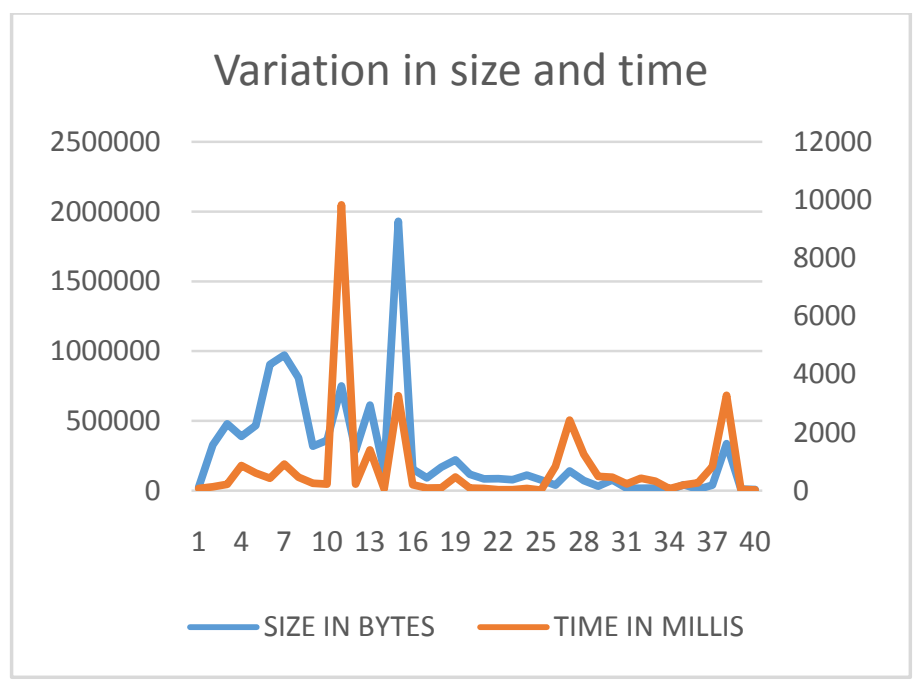

Fig4. Comparison between size of image and character recognition time

\subsection{PLATFORM FOR EVALUATION}

The platform utilized to evaluate the proposed approach includes a dual core CPU, the Intel Core 2 Duo with clock rate $2.0 \mathrm{GHz}$ and memory $4 \mathrm{~GB}$ DDR2 667 . MATLAB is used for simulation of code and verifying result.

\subsection{TEST SAMPLES OF IMAGES}

To validate our proposed approach we tested our assumptions and techniques on various images that were both noisy and noiseless. Some of them are listed below:

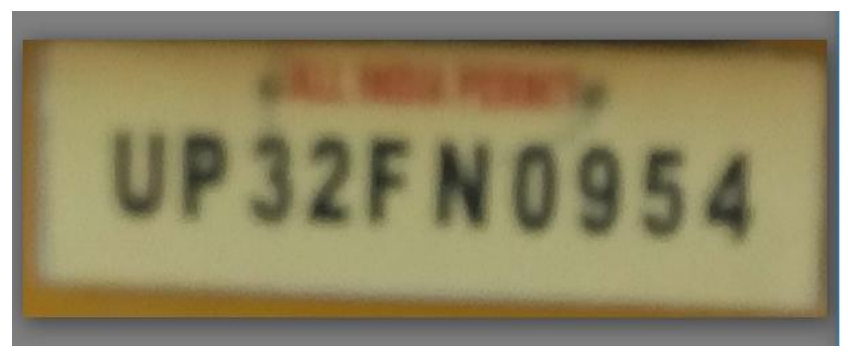

Fig.5. Blur image

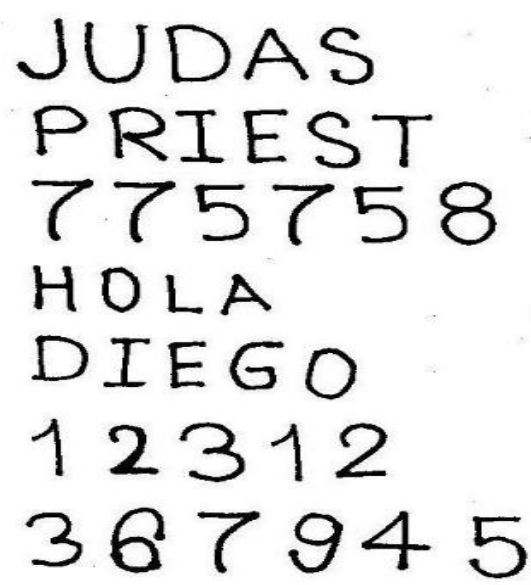

Fig.6. Noiseless grayscale image 


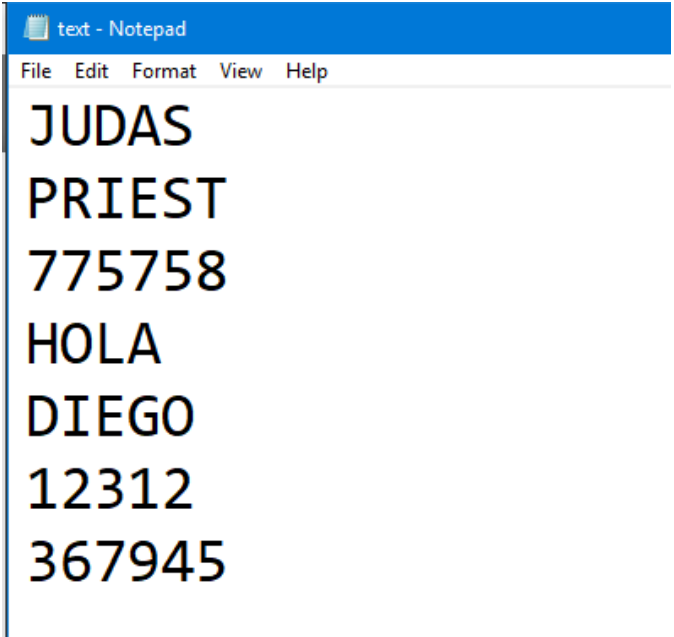

Fig.7. Output received of image in Fig. 6 after passing through OCR with AI

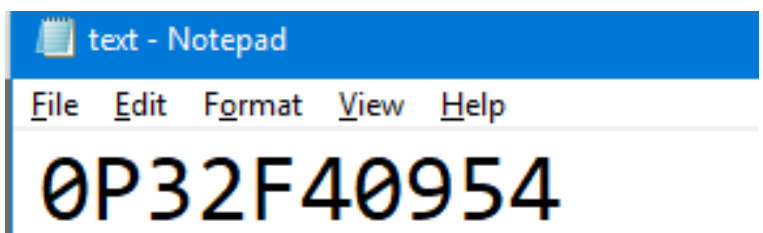

Fig.8 Output received of image in Fig. 5 after passing through OCR with AI

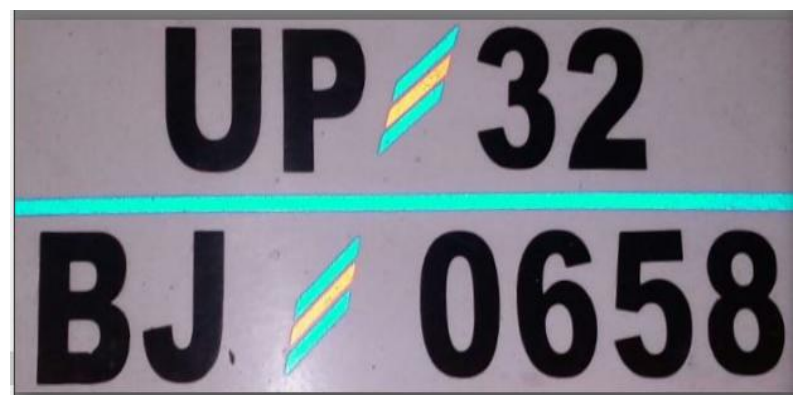

Fig.9. Noisy gray scale image

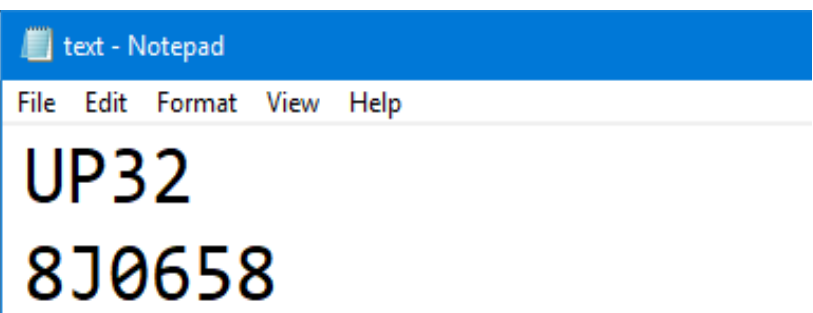

Fig.10 Output received of image in Fig. 9 after passing through OCR with AI

\section{CONCLUSION AND FUTURE SCOPE}

It was found that the method and approach that was used was able to recognize the character upto $100 \%$ when the image was noiseless and almost $95 \%$ in noisy images. It also helped in reducing the time for carrying out the whole procedure.
The future enhancements that can be done in this are that it can be trained for hand-written text. For better segmentation and character recognition we can implement the use of dictionary which will help in enhancing the performance of OCR.

\section{AKNOWLEDGEMENTS}

This work is supported by resources from Dr. Shalini Agarwal, Head of CS Department, Shri Ramswaroop Memorial University, Lucknow and Dr. A.K. Verma Director IOT Shri Ramswaroop Memorial University, Lucknow for promoting research activities in the Campus. We would also like to thank the anonymous reviewers for their significant and constructive critiques and suggestions, which substantially improved the quality of this paper.

\section{REFERENCES}

[1] Sameeksha Barve "Optical Character Recognition Using Artificial Neural Network" International Journal of Advanced Research in Computer Engineering \& Technology Volume 1, Issue 4, June 2012 ISSN: 2278 1323.

[2] R.Arnold, Poth Miklos" Character recognition using neural networks", Computational Intelligence and Informatics (CINTI), Hungary, pp 311-314 , 2010.

[3] Herbert F Schantz, "The history of OCR, optical character recognition", [Manchester Center, Vt.] Recognition Technologies Users Association, 1982.

[4] "Optical Character Recognition (OCR) for Printed Devnagari Script Using Artificial Neural Network" Raghuraj Singh, C. S. Yadav, Prabhat Verma, Vibhash YadavChaudhuri, A., Some Experiments on Optical Character Recognition Systems for different Languages using Soft Computing Techniques, Technical Report, Birla Institute of Technology Mesra, Patna Campus, India, 2010

[5] Isabelle Guyon, André Elisseeff, "An Introduction to Feature Extraction" in Studies in Fuzziness and Soft Computing, Springer pp 1-25 book series (STUDFUZZ, volume 207) 1995-2018.

[6] Rice, S. V., Nagy, G., Nartker, T. A., Optical Character Recognition: An Illustrated Guide to the Frontier, The Springer International Series in Engineering and Computer Science,Springer US, 1999

[7] Schantz, H. F., The History of OCR, Recognition Technology Users

[8] Association, Manchester Centre, VT, 1982.

[9] Mantas, J., An Overview of Character Recognition Methodologies, Pattern Recognition,19(6), pp 425-430, 1986.

[10] Rahul KALA, Harsh VAZIRANI, Anupam SHUKLA, and Ritu TIWARI "Offline Handwriting Recognition using Genetic Algorithm" International Journal of Computer Science Issues, Vol. 7, Issue 2, No 1, March 2010 ISSN (Online): 1694-0784, ISSN (Print): 1694-0814

[11] Brandon Maharrey "A Neural Network Implementation of Optical Character Recognition" Technical Report Number CSSE10-05 COMP 6600 - Artificial Intelligence Spring 2009 [8]. 
[12] Sang Sung Park, Won Gyo Jung, Young Geun Shin, Dong-Sik Jang "Optical Character Recognition System Using BP Algorithm" IJCSNS International Journal of Computer Sci 118 ence and Network Security, VOL.8 No.12, December 2008

[13] Made Edwin Wira Putra, Iping Supriana Suwardi "Structural off-line handwriting character recognition using approximate subgraph matching and levenshtein distance" International Conference on Computer Science and Computational Intelligence (ICCSCI 2015 Procedia Computer Science 59 ( 2015 ) 340 - 349

[14] Krupa Dholakia "A Survey on Handwritten Character Recognition Techniques For Various Indian Language" International Journal Of Computer Application (09758887) Volume 115-No. 1, April 2015

[15] Young, T. Y., Fu, K. S., Handbook of Pattern Recognition and Image Processing, Academic Press, 1986.

[16] Scurmann, J., Reading Machines, Proceedings of International Joint Conference on Pattern Recognition, Munich, pp 1031-1044, 1982.

[17] Arica, N., Vural, F. T. Y., An Overview of Character Recognition focused on Offline Handwriting, IEEE
Transactions on Systems, Man and Cybernetics - Part C: Applications and Reviews, 31(2), pp 216-233, 2001.

[18] Chowdhury Md Mizan, Tridib Chakraborty* and Suparna Karmakar "Text Recognition using Image Processing" International Journal of Advanced Research in Computer Science, Volume 8, No. 5, May - June 2017, ISSN No. 0976-5697.

[19] Sanjay Kumar, Narendra Sahu , Aakash Deep , Khushboo Gavel, Miss Rumi Ghosh "Offline Handwriting Character Recognition (for use of medical purpose)Using Neural Network" International Journal Of Engineering And Computer Science ISSN: 2319 7242,Volume 5 Issue 10 Oct. 2016, Page No. 1861218615 .

[20] Dr.Mrs.V.V.Patil , Rajharsh Vishnu Sanap , Rohini Babanrao Kharate "Optical Character Recognition Using Artificial Neural Network" International Journal of Engineering Research and General Science Volume 3, Issue 1, January-February, 2015 ISSN 2091-2730.

[21] Yusuf Khan, Kapil Kumar Gupta, Namrata Dhanda “ Optical Character Recognition using Intro Sort "International Journal of Computer Applications Volume 127-No.1,October 2015, PP 1-4. 